\title{
Science of Nursing and Health Practices
}

Science infirmière et pratiques en santé

\section{La rétroaction vidéo pour soutenir l'établissement de la relation mère-enfant en contexte d'allaitement maternel : une étude pilote}

\section{Video-Feedback Intervention to Support the Establishment of the Mother-Infant Relationship in Breastfeeding Mothers: A Pilot Study}

\author{
Linda Bell, Anne St-Pierre Pruneau, Marie Lacombe et Marjolaine Héon
}

Volume 3, numéro 1, 2020

URI : https://id.erudit.org/iderudit/1076472ar

DOI : https://doi.org/10.31770/2561-7516.1066

\section{Aller au sommaire du numéro}

\section{Éditeur(s)}

Réseau de recherche en interventions en sciences infirmières du Québec (RRISIQ)

\section{ISSN}

2561-7516 (numérique)

Découvrir la revue

Citer cet article

Bell, L., St-Pierre Pruneau, A., Lacombe, M. \& Héon, M. (2020). La rétroaction vidéo pour soutenir l'établissement de la relation mère-enfant en contexte d'allaitement maternel : une étude pilote. Science of Nursing and Health Practices / Science infirmière et pratiques en santé, 3(1), 1-15. https://doi.org/10.31770/2561-7516.1066

\section{Résumé de l'article}

Introduction : La rétroaction vidéo (RV) constitue une intervention efficace pour favoriser la sensibilité maternelle. La RV n’a pas été étudiée en contexte d'allaitement maternel (AM) auprès de mères vivant en contextes de vulnérabilité sociale et économique. Objectif : Examiner la faisabilité, l'acceptabilité et les effets préliminaires de la RV et de l'information de soutien à l'établissement de la relation mère-enfant dans le contexte d'AM auprès de mères participant à un programme communautaire. Méthodes : Une étude pilote à laquelle ont participé 20 mères, dont 6 dans le groupe $\mathrm{A}(2 \mathrm{RV}), 6$ dans le groupe $\mathrm{B}(1 \mathrm{RV})$ et 8 dans le groupe $\mathrm{C}$ (témoin), ainsi que 8 infirmières. Pendant la rétroaction, l'infirmière arrête la bande vidéo afin d'aider la mère à reconnaître une interaction positive entre elle et son enfant. Les participantes ont rempli un questionnaire d'acceptabilité et de faisabilité et participé à une entrevue. Les mères ont rempli un questionnaire sur la sensibilité maternelle et un autre sur l'allaitement. Résultats : Les participantes ont trouvé la RV acceptable et faisable. La RV n'a pas eu d'effet sur la sensibilité maternelle. La durée totale de l'allaitement semblait plus longue dans les groupes expérimentaux. De plus, les infirmières ont trouvé la RV utile pour renforcer la relation infirmière-mère. Discussion et conclusion : La RV est acceptable et faisable du point de vue des mères et des infirmières. Deux RV semblent plus satisfaisantes pour les mères qu'une seule. Plus d'études sont nécessaires pour évaluer l'efficacité de la RV.
Tous droits réservés @ C Linda Bell, Anne St-Pierre Pruneau, Marie Lacombe, Marjolaine Héon, 2020
Ce document est protégé par la loi sur le droit d'auteur. L'utilisation des services d’Érudit (y compris la reproduction) est assujettie à sa politique d'utilisation que vous pouvez consulter en ligne.

https://apropos.erudit.org/fr/usagers/politique-dutilisation/ 


\section{SCIENCE OF NURSING SCIENCE INFIRMIËRE}

\section{AND HEALTH PRACTICES ET PRATIQUES EN SANTÉ}

\section{La rétroaction vidéo pour soutenir l'établissement de la relation mère-enfant en contexte d'allaitement maternel : une étude pilote}

\section{Video-Feedback Intervention to Support the Establishment of the Mother-Infant Relationship in Breastfeeding Mothers: A Pilot Study}

Linda Bell, Inf., Ph. D., Université de Sherbrooke

Anne St-Pierre Pruneau, Inf., CSSS-IUGS, Université de Sherbrooke

Marie Lacombe, Inf., Ph. D., Université du Québec à Rimouski, Campus de Lévis

Marjolaine Héon, Inf., Ph. D., Université de Montréal

Correspondance | Correspondence:

Linda Bell, Inf., Ph. D.

Professeure titulaire

École des sciences infirmières

Faculté de médecine et des sciences de la santé

Université de Sherbrooke

3001, $12^{\mathrm{e}}$ Avenue Nord, Sherbrooke, QC, Canada, J1H 5N4

linda.bell@usherbrooke.ca 


Mots-clés
relation mère-
enfant;
allaitement
maternel;
intervention
rétroaction
vidéo

Introduction : La rétroaction vidéo (RV) constitue une intervention efficace pour favoriser la sensibilité maternelle. La RV n'a pas été étudiée en contexte d'allaitement maternel (AM) auprès de mères vivant en contextes de vulnérabilité sociale et économique. Objectif : Examiner la faisabilité, l'acceptabilité et les effets préliminaires de la RV et de l'information de soutien à l'établissement de la relation mère-enfant dans le contexte d'AM auprès de mères participant à un programme communautaire. Méthodes : Une étude pilote à laquelle ont participé 20 mères, dont 6 dans le groupe $A(2 \mathrm{RV}), 6$ dans le groupe $B(1 \mathrm{RV})$ et 8 dans le groupe $C$ (témoin), ainsi que 8 infirmières. Pendant la rétroaction, l'infirmière arrête la bande vidéo afin d'aider la mère à reconnaître une interaction positive entre elle et son enfant. Les participantes ont rempli un questionnaire d'acceptabilité et de faisabilité et participé à une entrevue. Les mères ont rempli un questionnaire sur la sensibilité maternelle et un autre sur l'allaitement. Résultats : Les participantes ont trouvé la RV acceptable et faisable. La RV n'a pas eu d'effet sur la sensibilité maternelle. La durée totale de l'allaitement semblait plus longue dans les groupes expérimentaux. De plus, les infirmières ont trouvé la RV utile pour renforcer la relation infirmièremère. Discussion et conclusion : La RV est acceptable et faisable du point de vue des mères et des infirmières. Deux RV semblent plus satisfaisantes pour les mères qu'une seule. Plus d'études sont nécessaires pour évaluer l'efficacité de la RV.

\footnotetext{
Abstract

Introduction: Video-feedback intervention (VFI) uses a strengths-based approach to enhance maternal sensitivity. VFI has been found to enhance maternal sensitivity, but has not been tested in the breastfeeding (BF) context with mothers in contexts of social and economical vulnerability. Objective: To assess the acceptability, feasibility and the preliminary effect of VFI combined with information on maternal sensitivity in a BF context with mothers enrolled in a community-based program. Methods: A pilot study was conducted. Twenty mothers and their babies were assigned either to group $A(2 \mathrm{VFI}, \mathrm{n}=6)$, group $B(1 \mathrm{VFI}, \mathrm{n}=6)$ or group $C$ (control, $n=8)$. Eight nurses participated. During VFI, the nurse would stop the video on significant sensitive interactions and help the mother acknowledge the positive exchanges taking place between her and her child. All participants completed acceptability and feasibility questionnaire and were interviewed. Mothers completed a maternal sensitivity scale and a BF questionnaire. Results: All participants found VFI acceptable and feasible. The VFI had no effect on maternal sensitivity. Total BF duration seemed longer in the intervention groups. Also, the nurses found the VFI helpful to strengthen the nursemother relationship. Discussion and conclusion: VFI is feasible and acceptable for mothers and nurses. Two VFI seemed to be more satisfying to mothers than one. More research is needed to determine the effectiveness of VFI.
}

\section{Keywords}

mother-infant relationship; breastfeeding; video-feedback intervention 


\section{INTRODUCTION}

La qualité de la relation que l'enfant développe avec ses parents au cours des premiers mois de sa vie, particulièrement avec sa mère s'il est allaité, constitue la pierre angulaire de sa croissance et de son développement (Bowlby, 1984). C'est dans le cadre d'une relation empreinte de sensibilité que l'enfant apprend à réguler ses besoins et ses émotions, à se sentir apaisé et sécurisé (Cassidy, 1999). Les enfants et leur mère recevant des services dans le cadre du programme Services intégrés en périnatalité et en petite enfance (SIPPE) présentent des caractéristiques personnelles et sociales les rendant plus à risque de développer des difficultés relationnelles. Les critères d'admissibilité au programme incluent la sous-scolarisation et le faible niveau socioéconomique (ministère de la Santé et des Services sociaux du Québec [MSSSQ], 2019). Pour leur part, les enfants peuvent poser des défis à l'établissement de la relation, notamment en raison de leur tempérament ou de difficultés à signaler clairement leurs besoins, spécialement en contexte d'allaitement. Les infirmières sont les principales intervenantes dans le cadre des suivis SIPPE au cours de la première année de vie de l'enfant, ce qui les place en situation de favoriser l'établissement de la relation entre la mère et son enfant. De plus, les infirmières du programme SIPPE accordent un soutien particulier à la mère qui allaite.

L'allaitement maternel est reconnu non seulement comme un mode d'alimentation, mais aussi comme un contexte interactionnel favorable à l'établissement de la relation mère-enfant (Jansen et al., 2008; Peñacoba et Catala, 2019). L'allaitement répond aux besoins de chaleur, de nourriture, de succion et de contact physique du bébé. II permet des échanges entre la mère et l'enfant et suscite la proximité physique et des échanges affectifs. Les connaissances actuelles soutiennent l'hypothèse selon laquelle la sensibilité maternelle, qui est définie comme étant la détection des comportements, l'interprétation juste et une réponse juste et appropriée aux besoins de l'enfant dans un délai raisonnable (de Woolf et van IJzendoorn, 1997), est associée à la poursuite de l'allaitement (Britton et al., 2006). Selon Kronborg et ses collègues (2015), l'allaitement maternel entraîne une perception de soi et des émotions positives chez les mères. Pour leur part, les enfants ayant été allaités pour une période d'au moins six mois seraient plus susceptibles de développer des compétences socioémotionnelles (Turner et al., 2019) et un attachement sécurisant (Tharner et al., 2012).

Le modèle de l'établissement de la relation mère-enfant a été développé dans la perspective d'apprécier la qualité et de promouvoir l'établissement de la relation mère-enfant (Bell et al., 2004b; Bell et al., 2007; Bell, 2008). Selon ce modèle, la relation mère-enfant est constituée de cinq composantes qui chacune est influencée par le niveau de sensibilité maternelle présent dans la relation (voir Figure 1). Ces composantes sont : la découverte de l'enfant, le contact affectif, la proximité physique, la communication et l'engagement dans le rôle parental. Ce modèle a été validé auprès de mères de nouveau-nés (Doyon, 2010), de mères et de pères d'enfants prématurés (Martel et al., 2011), en contexte d'allaitement maternel (Bell et al., 2012; Dubé et al., 2019) et de dépression postnatale (Couture et al., 2019; Courtois et al., 2012). Il a également servi au développement d'interventions infirmières ayant pour but de soutenir l'établissement de la relation mère-enfant, notamment le massage de l'enfant prématuré (Larone Juneau, 2013) et le positionnement thérapeutique des enfants nés prématurément (Lavallée et al., 2019).

La rétroaction vidéo est une approche qui consiste à observer et à discuter des interactions mère-enfant préalablement saisies sur bande vidéo (Juffer et al., 2008). Les données actuelles suggèrent qu'une intervention brève de rétroaction vidéo, soit moins de cinq interventions, constitue une intervention efficace pour favoriser la sensibilité maternelle et un attachement sécurisant chez l'enfant (Bakermans-Kranenburg et al., 2003; Fukkink, 2008), particulièrement dans les familles présentant des facteurs de risque à l'établissement de la relation mère-enfant (Bakermans-Kranenburg et al., 2003). L'efficacité de l'intervention par rétroaction vidéo pour soutenir la relation parents-enfant a été étudiée en contextes de problèmes de comportement chez 
l'enfant (lles et al., 2017), de prématurité (Hoffenkamp et al., 2015), de problèmes d'alimentation (Stein et al., 2006), de la maltraitance de l'enfant (Leclère et al., 2018), des enfants présentant des problèmes autistiques (Poslawsky et al., 2014), des mères de minorité ethnique (Yagmur, et al., 2014), des clientèles vulnérables (Juffer et Bakermans-Kranenburg, 2018), des parents présentant des déficiences intellectuelles (Hodes et al., 2018) ainsi qu'auprès d'enfants adoptés (Schoemaker et al., 2018). Cependant, son efficacité à soutenir la relation mère-enfant dans le contexte de l'allaitement maternel n'a pas été étudiée jusqu'à maintenant.

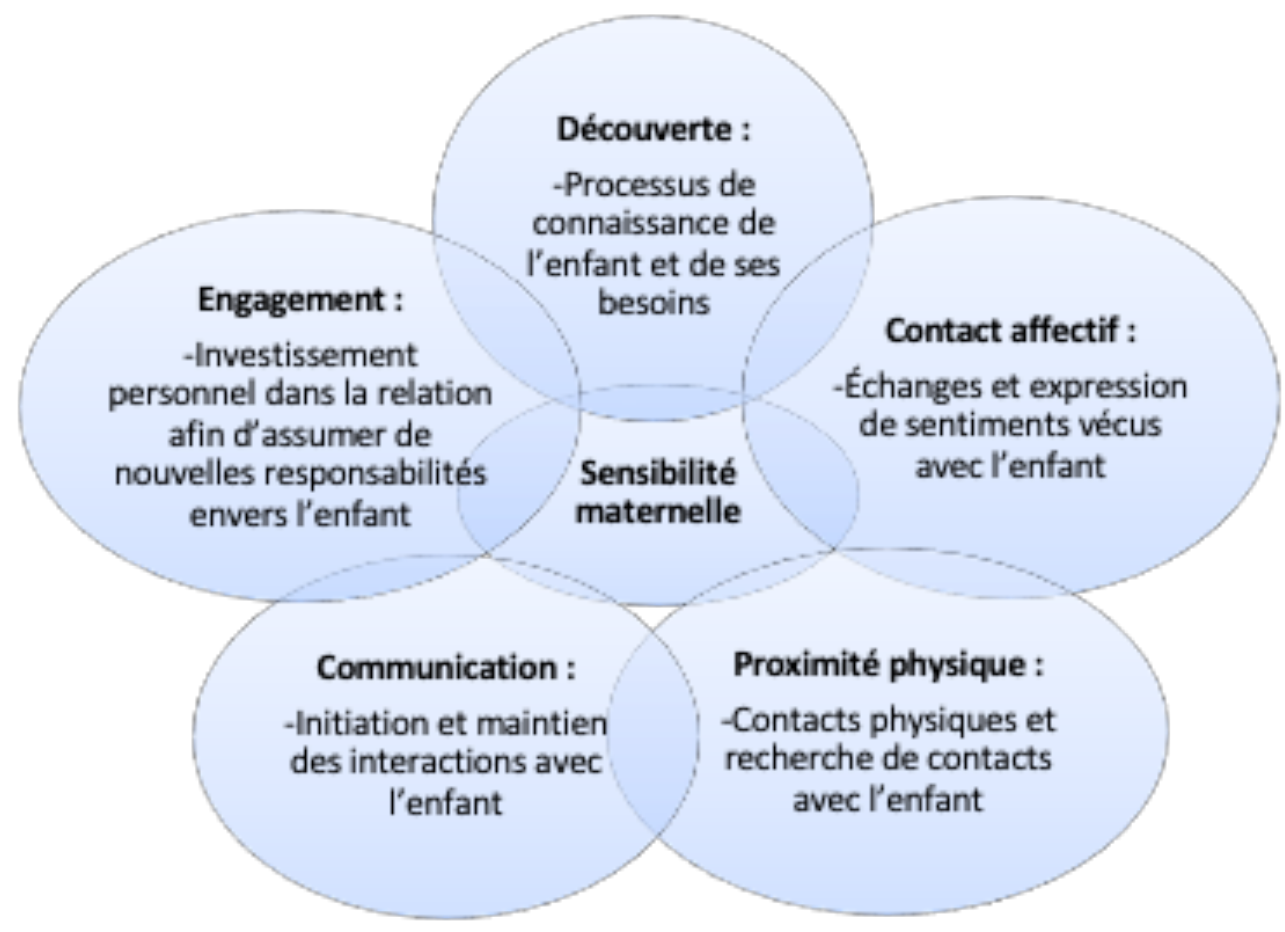

Figure 1. Modèle de l'établissement de la relation mère-enfant (Bell, 2008)

\section{OBJECTIF}

La présente étude a pour but d'examiner la faisabilité, l'acceptabilité et les effets préliminaires d'une intervention de soutien à l'établissement de la relation mère-enfant en utilisant la rétroaction vidéo et de l'information sur l'établissement de la relation mère-enfant dans le contexte de l'allaitement maternel auprès de mères suivies dans le programme SIPPE. Les objectifs spécifiques sont de : 1) décrire la faisabilité du recrutement des mères et des infirmières pour cette intervention, 2) décrire l'acceptabilité et la faisabilité de l'intervention selon les mères, 3) décrire l'acceptabilité et la faisabilité de l'intervention selon les infirmières et 4 ) décrire les effets préliminaires de l'intervention sur la sensibilité maternelle et sur la poursuite de l'allaitement à trois mois.

MÉTHODES

DEVIS

Il s'agit d'un essai clinique randomisé pragmatique permettant d'évaluer la faisabilité, l'acceptabilité et les effets préliminaires de l'approche de rétroaction vidéo dans le soutien à l'établissement de la relation mère-enfant en contexte d'allaitement. Une approche pragmatique, qui consiste à évaluer une 
intervention auprès d'une variété de participants en tenant compte des caractéristiques de l'environnement clinique, a été privilégiée (Oxman et al., 2009; Zwarenstein et Treweek, 2009).

\section{ÉCHANTILLON ET PROCÉDURE}

Les mères et leur enfant ont été recrutés dans un Centre de Santé et de Services sociaux (CSSS) de la région de l'Estrie où plus de 465 familles reçoivent des services dans les programmes SIPPE. Pour participer, les mères devaient répondre aux critères suivants : être âgée de 18 ans et plus, être en bonne santé, recevoir des services dans les programmes SIPPE dès la période prénatale ainsi que lire et parler le français. Les nourrissons éligibles devaient, pour leur part, être nés à terme, être en bonne santé et être âgés de quatre mois et moins. Nous avons exclu les dyades mères-enfant déjà connues pour des problèmes de négligence ou d'abus (Loi de la protection de la jeunesse) ainsi que les mères de jumeaux ou triplets.

Deux semaines suivant la naissance de l'enfant, les participantes étaient invitées à compléter le questionnaire sociodémographique et à choisir parmi trois enveloppes scellées contenant le résultat de la randomisation (Groupe $A, B$ ou $C$ ). Les mères du groupe expérimental ont reçu deux interventions (Groupe $A, n=6$ ) ou une intervention (Groupe $B, n=6$ ). Les mères du groupe témoin (Groupe $C, n=8$ ) ont reçu les soins habituels de la part de leur infirmière.

Pour leur part, les infirmières ont été recrutées dans le cadre d'une rencontre avec la chercheuse principale. La rencontre visait à présenter l'étude, déterminer l'implication attendue des infirmières et obtenir la collaboration des gestionnaires. Pour être éligibles à participer à l'étude, les infirmières devaient avoir suivi la formation sur la rétroaction vidéo offerte par l'Équipe régionale de formation en attachement parents-enfant basée sur le modèle de l'établissement de la relation parents-enfant utilisé dans la présente étude.

L'étude a reçu l'approbation du comité éthique de la recherche des CSSS de l'Estrie. Les participantes ont signé un formulaire de consentement leur assurant la confidentialité et suggérant qu'elles pouvaient se retirer en tout temps de l'étude ou ne pas répondre à des questions.

\section{INTERVENTION DE RÉTROACTION VIDÉO SUR LA RELATION MÈRE-ENFANT EN CONTEXTE D'ALLAITEMENT MATERNEL}

La rétroaction vidéo est une approche consistant à observer et discuter des interactions saisies sur bande vidéo. Elle est basée sur une approche cognitivo-comportementale ayant pour but de favoriser la sensibilité parentale (Juffer et al., 2008).

Dans le cadre de la présente étude, le déroulement de l'approche se résume en trois étapes, soit : 1) saisir l'interaction parents-enfant sur bande vidéo, 2) analyser la bande vidéo et préparer le retour avec la mère, et 3 ) effectuer le retour avec la mère.

Premièrement, l'infirmière saisit sur bande vidéo une interaction mère-enfant en contexte d'allaitement, au domicile de la mère. Durant cette phase, l'infirmière adopte une position d'observatrice et $n$ 'intervient pas dans l'interaction entre la mère et son bébé. Dans le contexte de la présente étude, un groupe a reçu une RV et un autre en a reçu deux, dans le but d'estimer la faisabilité et l'acceptabilité de plus d'une intervention ainsi que sa valeur ajoutée.

Dans un deuxième temps, l'infirmière visionne la bande vidéo dans la perspective d'apprécier la qualité des interactions entre la mère et son enfant et de planifier le retour avec la mère. L'évaluation de la qualité de la sensibilité maternelle est faite à l'aide du modèle de l'établissement de la relation mère-enfant (Bell, 2008). Cette étape a été réalisée avec une experte dans I'utilisation du modèle, ayant reçu la formation sur l'attachement parentsenfant et occupant une fonction de conseillère clinique au sein de l'établissement. Celle-ci a contribué à enrichir l'observation et à planifier le retour auprès du parent. Plus précisément, $c^{\prime}$ est en collaboration avec l'infirmière que l'experte du modèle a ciblé une séquence vidéo pertinente, c'est-à-dire qui favorise l'atteinte du but visé, qui est d'aider la mère à mieux connaître son enfant et à se sentir compétente. Elle a également aidé I'infirmière à planifier un retour constructif auprès du parent. Cette étape est importante pour 
favoriser la confiance de l'intervenante, mais également pour atteindre les buts visés par l'intervention (Fukkink, 2008).

La troisième étape consiste à faire le retour sur la bande vidéo avec la mère. Ce retour a eu lieu dans les deux semaines suivant la saisie de l'interaction sur bande vidéo. Avant de débuter le visionnement, l'infirmière explique la procédure, clarifie les objectifs et favorise l'expression des attentes et des craintes de la mère. Le fait de positionner clairement l'objectif de l'intervention comme étant un travail de collaboration entre l'infirmière et la mère, visant à favoriser l'allaitement maternel et la relation mère-enfant, est crucial pour favoriser le plein engagement de la mère dans la démarche et la décentrer du jugement sur ses propres comportements. Durant le retour avec la mère, la bande vidéo est arrêtée à différents moments afin de mettre l'emphase sur une interaction positive et renforcer la mère dans ses capacités à reconnaître les signes émis par son enfant et y répondre adéquatement. La rétroaction positive est ancrée dans l'observation du comportement de l'enfant en réaction à celui de la mère de sorte que la mère devienne son propre modèle et développe un sentiment de compétence dans l'interaction avec son enfant.

St-Laurent et ses collaborateurs (2008), ayant utilisé et évalué ce type d'intervention, estiment que toute intervention ayant pour effet de dévaloriser le parent agit à l'encontre de cette approche. La rétroaction vidéo est utilisée comme une modalité d'intervention à court terme. On estime que l'efficacité de cette approche tient à la qualité du retour fait par le clinicien, bien sûr, mais tout autant au fait que cette procédure donne l'occasion au parent de se voir en action avec son enfant et d'observer attentivement, de manière plus objective, les comportements de son enfant. D'une certaine façon, le parent est son propre modèle de changement, puisqu'il peut prendre le temps de s'observer dans l'action, ce qui est impossible autrement (Zelenko et Benham, 2000).

Enfin, un dépliant contenant de l'information sur la sensibilité maternelle et l'attachement parents-enfant a été remis à la mère. Celui-ci a été développé par l'Équipe régionale de formation en attachement parents-enfant. II vise à informer les mères sur l'expression de la sensibilité maternelle dans les cinq composantes de la relation de même que la contribution de l'enfant à l'établissement de la relation, ainsi que les facteurs pouvant influencer la relation. Les types d'attachement, sécurisant et insécurisant, découlant de la qualité des interactions sont également présentés. Ce dépliant a été testé auprès de mères ayant des caractéristiques semblables à celles qui ont participé à la présente étude.

\section{OUTILS DE COLLECTE DES DONNÉES}

Acceptabilité et faisabilité selon les mères. $\mathrm{Au}$ cours des deux semaines suivant la/les rétroaction(s) vidéo, l'acceptabilité et la faisabilité de l'intervention selon le point de vue des mères ont été documentées à l'aide d'un questionnaire auto-administré et d'une entrevue semi-dirigée réalisée par l'assistante de recherche au domicile de la mère. Le questionnaire comprend huit questions avec une échelle de réponse de type Likert graduée de 1 à 5 (" pas du tout en accord " à " tout à fait d'accord "). Le score attendu d'acceptabilité et de faisabilité de l'intervention de rétroaction vidéo en contexte d'allaitement était de $80 \%$ (plutôt d'accord, tout à fait d'accord). Le questionnaire a été prétesté auprès de quatre mères. L'entrevue semi-dirigée, d'une durée de 20 minutes, a permis d'obtenir le point de vue des mères sur l'efficacité perçue ainsi que les retombées potentielles (négatives ou positives) de l'intervention. Le guide d'entrevue, comprenant quatre questions ouvertes, a été prétesté auprès de quatre mères.

Acceptabilité et faisabilité selon les infirmières. Le point de vue des infirmières sur l'acceptabilité et la faisabilité de l'intervention a été sondé à l'aide d'un questionnaire autoadministré et d'une entrevue semi-dirigée. Le questionnaire, rempli dans les deux semaines suivant l'intervention, comprend neuf questions avec une échelle de réponse de type Likert graduée de 1 à 5 (" pas du tout en accord " à " tout à fait d'accord "). Le score attendu d'acceptabilité et de faisabilité de l'intervention de rétroaction vidéo en contexte d'allaitement était de $80 \%$ (plutôt d'accord, tout à fait d'accord). L'entrevue semidirigée d'une durée de 20 minutes visait à décrire I'intervention réalisée ainsi que les barrières et les facilitateurs à la réalisation de la rétroaction vidéo. 
Effets préliminaires de l'intervention de rétroaction vidéo. Les effets de l'intervention sur la sensibilité maternelle et sur la poursuite de l'allaitement ont été documentés auprès des mères par le biais de deux questionnaires autoadministrés. Premièrement, avant et après l'intervention, les mères ont rempli un questionnaire comprenant des données sur l'allaitement maternel, tel que le type d'allaitement (maternel exclusif ou avec complément), la durée, le soutien reçu, l'objectif et les difficultés rencontrées. Deuxièmement, avant et après l'intervention, les mères ont complété l'Échelle de la sensibilité maternelle (ÉSM; Bell et al., 2004a) qui est composée de 33 énoncés. L'ÉSM comprend cinq sous-échelles : la découverte, le contact affectif, l'engagement, le contact physique et la communication. Les énoncés sont cotés sur une échelle de Likert en cinq points variant de "tout à fait d'accord " à " tout à fait en désaccord ». Six items sont formulés négativement. Plus le score sur l'instrument est faible, plus la sensibilité maternelle de la mère est élevée. La consistance interne de l'instrument de mesure pour les sous-échelles varie entre 0,54 et 0,83 (Bell et al., 2004a). La validité de contenu et la cohérence interne de l'échelle ont été testées dans le cadre d'études menées auprès de mères ayant un bébé âgé de moins de six mois (Bell et al., 2012; Courtois et al., 2012; Doyon, 2010; Dubé et al., 2019; Lavallée et al., 2019; Martel et al., 2011). Dans le cadre de la présente étude, l'ÉSM a été complétée par les mères avant et après l'intervention dans tous les groupes.

Le Tableau 1 résume la séquence de la collecte des données.

\section{Tableau 1}

Collecte des données

\begin{tabular}{lcc}
\multicolumn{1}{c}{ Données collectées } & Pré Int & Post Int \\
\hline MÈRES & $\checkmark$ & $\checkmark$ \\
\hline Échelle de sensibilité maternelle (ÉSM, Bell et al., 2004) & $\checkmark$ & $\checkmark$ \\
Questionnaire sur l'allaitement maternel & $\checkmark$ & $\checkmark$ \\
Questionnaire sociodémographique & $\checkmark$ \\
Questionnaire acceptabilité et faisabilité & \\
Entrevue semi-structurée sur l'acceptabilité, la faisabilité et les effets perçus de & \\
l'intervention & $\checkmark$ \\
\hline INFIRMIÈRES & $\checkmark$ \\
\hline Questionnaire sociodémographique & $\checkmark$ \\
Questionnaire acceptabilité et faisabilité \\
Entrevue semi-structurée sur l'acceptabilité, la faisabilité et les effets perçus de \\
l'intervention
\end{tabular}

\section{ANALYSES}

Des analyses descriptives ont été effectuées telles que la moyenne et l'écart-type, la médiane et l'étendue interquartile, la fréquence et la proportion, dépendamment de la distribution des données. La comparaison entre les groupes a été effectuée à l'aide d'analyses bivariées appropriées selon le type de données, soit des tests non paramétriques de Kruskall-Wallis, un test exact de Fisher pour les variables de type catégorique ou un test de Mann-Whitney. Les données qualitatives ont été analysées à l'aide d'une codification du contenu, telle que décrite par L'Écuyer (1990). II s'agit d'une méthode d'analyse orientée vers la recherche de signification dont la procédure vise à estimer le poids des idées soulevées ainsi que leur pertinence en fonction du modèle utilisé. Il s'agit d'une procédure d'analyse réalisée essentiellement en cinq étapes, soit :

1. la saisie des données

2. la codification des données

3. la validation inter-juges de la codification des données

4. l'analyse des similarités et le développement des catégories

5. la saturation des données. 


\section{DONNÉES SOCIODÉMOGRAPHIQUES}

Le Tableau 2 présente les données sociodémographiques des mères $(n=20)$, des bébés $(n=20)$ ainsi que des infirmières $(n=8)$ ayant participé à l'étude.

\section{FAISABILITÉ DU RECRUTEMENT ET DE LA COLLECTE DES DONNÉES}

Le recrutement a eu lieu sur une période de neuf mois. Durant cette période, 29 mères répondaient aux critères d'éligibilité de l'étude. Parmi celles-ci, six ont refusé de participer et trois ont été exclues. Les raisons des mères ayant refusé de participer se décrivent comme suit : une mère pour motif religieux, trois mères réticentes à être filmées durant l'allaitement, une mère manquait de temps et une mère n'a pas donné de raison. Trois mères ayant accepté de participer en

prénatal ont été exclues en postnatal, dont deux mères ayant décidé de ne pas allaiter et un bébé présentant un problème de santé. Au total, 20 mères ont été recrutées avec leur enfant âgé entre 10 jours et quatre mois.

Les 12 infirmières travaillant dans le programme SIPPE ont accepté de participer à l'étude. De celles-ci, huit ont réalisé l'intervention auprès des mères de leur clientèle en suivi SIPPE et qui avaient accepté de participer. Quatre d'entre elles ont réalisé deux interventions. La collecte des données a eu lieu comme prévu, avec les 20 mères filmées en interaction avec leur enfant pour une durée de 15 minutes durant l'allaitement. Toutes les bandes vidéo ont été visionnées et analysées en collaboration avec une experte du modèle de l'établissement de la relation mère-enfant et tous les retours avec la mère ont eu lieu dans la semaine suivant la saisie sur bande vidéo. Pour le groupe $A$, une deuxième séance de rétroaction vidéo, incluant les trois étapes décrites ci-haut, a eu lieu deux semaines après la première.

Tableau 2

Données sociodémographiques des participants

\begin{tabular}{|c|c|c|c|c|c|c|}
\hline & $\begin{array}{c}\text { Groupe A } \\
(n=6)\end{array}$ & $\begin{array}{c}\text { Groupe B } \\
(n=6)\end{array}$ & $\begin{array}{c}\text { Groupe } A+B \\
\quad(n=12)\end{array}$ & $\begin{array}{c}\text { Groupe C } \\
(n=8)\end{array}$ & $\begin{array}{c}\text { Valeur } p \\
\text { A, B, C }\end{array}$ & $\begin{array}{l}\text { Valeur } p \\
A+B \text { vs C }\end{array}$ \\
\hline \multicolumn{7}{|l|}{ MÈRES $(n=20)$} \\
\hline Âge (années) & 33,5 & 25,5 & 30,0 & 29,5 & 0,253 & 0,373 \\
\hline - Med. (É́) & $(27,0-34,3)$ & $(20,8-32,3)$ & $(21,5-34,0)$ & $(20,8-31,5)$ & & \\
\hline \multicolumn{7}{|l|}{ Éducation } \\
\hline$<12$ ans & $2(33,3)$ & $5(83,3)$ & $7(58,3)$ & $6(75,0)$ & 0,143 & 0,642 \\
\hline$>12$ ans & $4(66,7)$ & $1(16,7)$ & $5(41,7)$ & $2(25,0)$ & & \\
\hline \multicolumn{7}{|l|}{ Revenu ${ }^{a}$} \\
\hline $10-49,000$ & $6(100)$ & $6(100)$ & $12(100)$ & $7(87,5)$ & 0,454 & 0,400 \\
\hline $50-99,000$ & $0(0)$ & $0(0)$ & $0(0)$ & $1(12,5)$ & & \\
\hline \multicolumn{7}{|l|}{ Pays de naissance } \\
\hline Canada & $1(16,7)$ & $5(83,3)$ & $6(50,0)$ & $6(75,0)$ & 0,010 & 0,373 \\
\hline Autre pays & $5(83,3)$ & $1(16,7)$ & $6(50,0)$ & $2(25,0)$ & & \\
\hline \multicolumn{7}{|l|}{ Langue parlée } \\
\hline Français & $2(33,3)$ & $5(83,3)$ & $7(58,3)$ & $8(100)$ & 0,015 & 0,055 \\
\hline Autre langue & $4(66,7)$ & $1(16,7)$ & $5(41,7)$ & $0(0)$ & & \\
\hline
\end{tabular}




\begin{tabular}{|c|c|c|c|c|c|c|}
\hline & $\begin{array}{c}\text { Groupe A } \\
(n=6)\end{array}$ & $\begin{array}{c}\text { Groupe B } \\
(n=6)\end{array}$ & $\begin{array}{l}\text { Groupe } A+B \\
(n=12)\end{array}$ & $\begin{array}{c}\text { Groupe C } \\
(n=8)\end{array}$ & $\begin{array}{c}\text { Valeur } p \\
\text { A, B, C }\end{array}$ & $\begin{array}{l}\text { Valeur } p \\
\text { A+B vs C }\end{array}$ \\
\hline \multicolumn{7}{|l|}{ BÉBÉS ( $n=20$ ) } \\
\hline $\begin{array}{l}\text { Poids du bébé } \\
\text { (kg) - Méd. (Él) }\end{array}$ & $\begin{array}{c}3,6 \\
(3,4-3,7)\end{array}$ & $\begin{array}{c}3,8 \\
(2,7-28,1)\end{array}$ & $\begin{array}{c}3,6 \\
(3,4-4)\end{array}$ & $\begin{array}{c}3,1 \\
(2,8-3,3)\end{array}$ & 0,107 & 0,035 \\
\hline $\begin{array}{l}\text { Âge gestationnel } \\
\text { (jours) - Méd. (Él) }\end{array}$ & $\begin{array}{c}281 \\
(274,8-287,5)\end{array}$ & $\begin{array}{c}268,4 \\
(264,3-273,3)\end{array}$ & $\begin{array}{c}274 \\
(268-281,5)\end{array}$ & $\begin{array}{c}272 \\
(263-277,5)\end{array}$ & 0,047 & 0,333 \\
\hline $\begin{array}{l}\text { Âge du bébé (jours) } \\
\text { au moment de l'int. }\end{array}$ & $\begin{array}{c}16,5 \\
(11-32)\end{array}$ & $\begin{array}{c}18,0 \\
(14-32)\end{array}$ & $\begin{array}{c}21,5 \\
(11-32)\end{array}$ & $\begin{array}{c}30,0 \\
(8-57)\end{array}$ & & 0,898 \\
\hline
\end{tabular}

INFIRMIÈRES $(n=8)$

\begin{tabular}{ll}
\hline Âge & $<40$ ans $=3(37,5 \%)$ \\
& $>40$ ans $=5(62,5 \%)$ \\
Statut & Temps complet $=6(75 \%)$ \\
& Temps partiel $=2(25 \%)$ \\
Années & $<20$ ans $=5(62,5 \%)$ \\
d'expérience & $>20$ ans $=3(37,5 \%)$ \\
Années & $<10$ ans $=8(100 \%)$ \\
d'expérience SIPPE &
\end{tabular}

Méd.: Médiane, Él: Écart interquartile (25e-75e percentile)

a Dollars canadiens

\section{ACCEPTABILITÉ, FAISABILITÉ ET EFFETS PRÉLIMINAIRES DE L'INTERVENTION SELON LES MÈRES}

Le Tableau 3 présente les résultats concernant l'acceptabilité et la faisabilité de I'intervention de rétroaction vidéo en contexte $\mathrm{d}$ 'allaitement. Toutes les mères (Groupes $\mathrm{A}$ et $\mathrm{B}=$ $100 \%$ ) ont trouvé acceptable d'être filmées durant un allaitement et aucune ne s'est sentie jugée durant l'intervention (Groupes A et B $=100 \%$ ). La majorité des mères estime avoir participé pour une raison autre que celle de faire plaisir à l'infirmière (Groupes A et B $=83,3 \%$ ) et s'est sentie respectée durant la procédure (Groupe $A=100 \%$ et Groupe $B=83,3 \%)$. La majorité des mères du groupe ayant reçu deux interventions (Groupe $A=83,3 \%$ ) ont jugé la rétroaction de l'infirmière utile alors que ce taux est de $66,6 \%$ chez les mères ayant reçu une seule séance de rétroaction vidéo. Se voir en interaction avec leur enfant durant l'allaitement a été jugé utile pour plus de $80 \%$ des mères, indépendamment du groupe. Toutes les mères (100 \%), indépendamment du groupe, sont satisfaites de l'intervention et la majorité la recommanderait (Groupe $A=83,3 \%$, Groupe B = $100 \%$ ). En somme, les seuils d'acceptabilité et de faisabilité de l'intervention, préalablement fixés à $80 \%$, ont été en majorité obtenus, sauf en ce qui concerne l'utilité de la rétroaction de l'infirmière dans le groupe ayant reçu une intervention seulement (Groupe B $=66,6 \%$ ).

Selon les mères, les retombées de la rétroaction vidéo sont : 1) d'observer des interactions avec leur enfant, 2) de prendre conscience des points positifs et négatifs dans l'interaction avec leur enfant, 3) d'obtenir une rétroaction positive de l'infirmière, 4) d'avoir une meilleure conscience des effets de leur comportement sur l'enfant, 5) de remarquer quelque chose de nouveau (observer une nouveauté) dans la relation avec l'enfant et, 6) de mieux comprendre les besoins de leur enfant. Aucune mère n'a soulevé d'aspects négatifs à l'intervention. Voir le Tableau 4 pour des extraits du verbatim caractéristiques de chacune des retombées soulevées par les mères. 
Tableau 3

Acceptabilité et faisabilité de la rétroaction vidéo selon les mères $(n=12)$

\begin{tabular}{llcc}
\hline \multicolumn{1}{c}{ Items du questionnaire } & \multicolumn{1}{c}{$\begin{array}{c}\text { Groupe A } \\
\mathbf{n}(\%)\end{array}$} & $\begin{array}{c}\text { Groupe B } \\
\mathbf{n}(\%)\end{array}$ \\
\hline Être filmée durant l'allaitement est acceptable & Tout à fait d'accord & $6(100)$ & $6(100)$ \\
\hline S'est sentie jugée & Pas du tout en accord & $6(100)$ & $6(100)$ \\
\hline La rétroaction de l'infirmière est utile & D'accord & $0(0)$ & $2(33,3)$ \\
\cline { 2 - 4 } & Tout à fait d'accord & $5(83,3)$ & $2(33,3)$ \\
\hline $\begin{array}{l}\text { A participé à l'étude pour faire plaisir à } \\
\text { l'infirmière }\end{array}$ & Pas du tout en accord & $5(83,3)$ & $5(83,3)$ \\
\hline $\begin{array}{l}\text { Le comportement de l'infirmière est } \\
\text { respectueux }\end{array}$ & Tout à fait d'accord & $6(100)$ & $5(83,3)$ \\
\hline $\begin{array}{l}\text { L'expérience de se voir en interaction avec son } \\
\text { bébé sur bande vidéo est utile }\end{array}$ & D'accord & $2(33,3)$ & $3(50)$ \\
\cline { 2 - 4 } & Tout à fait d'accord & $3(50)$ & $2(33,3)$ \\
\hline Satisfaite du résultat & D'accord & $1(16,7)$ & $2(33,3)$ \\
\cline { 2 - 4 } & Tout à fait d'accord & $5(83,3)$ & $4(66,7)$ \\
\hline $\begin{array}{l}\text { Recommanderait l'intervention de rétroaction } \\
\text { vidéo }\end{array}$ & D'accord & $0(0)$ & $1(16,7)$ \\
\cline { 2 - 4 } & Tout à fait d'accord & $5(83,3)$ & $5(83,3)$ \\
\hline
\end{tabular}

\section{Tableau 4}

Perception de l'effet de la rétroaction vidéo selon les mères $(n=12)$

1. Observer des interactions mère-enfant $(n=10)$

" On voyait vraiment qu'il y avait une connexion entre nous deux, qu'elle me comprenait, que je la comprenais, même sans qu'elle parle, avec ses gestes, comment elle réagissait. J'ai vu que j'ai une bonne relation avec mon bébé. » (M36)

2. Prendre conscience des points positifs et négatifs dans l'interaction avec l'enfant $(n=10)$

" C'est un choc de se voir avec notre bébé et de se demander : est-ce vraiment ce que je veux lui offrir? Tu dois te poser des questions à propos de ta relation avec ton bébé. » (M43)

3. Obtenir un feedback positif de l'infirmière $(n=9)$

"La rétroaction vidéo nous apporte du confort, de la confiance. Je pouvais voir les choses que je faisais, cela peut aider les mères à observer ce qu'elles font de bien. " (M42)

4. Meilleure conscience des effets de son comportement sur l'enfant $(n=7)$

" [J'ai réalisé] que je faisais du bon travail, que j'étais bonne dans ce que je faisais, que j'étais capable de répondre aux besoins de mon bébé. » (M22)

5. Remarquer quelque chose de nouveau dans la relation avec l'enfant $(n=5)$

" Je pouvais voir à quel point je suis confortable avec mon bébé. Tu ne remarques pas ces choses-là ; c'est comme une habitude. Quand j'allaite, elle place sa petite main sur moi. Jamais je n'avais remarqué ça avant. » (M36)

6. Augmenter la compréhension des besoins de son enfant $(n=4)$

"La vidéo m'a permis de mieux comprendre les besoins de mon enfant. » (M24) 


\section{ACCEPTABILITÉ ET FAISABILITÉ DE L'INTERVENTION SELON LES INFIRMIÈRES}

Le Tableau 5 présente les résultats concernant l'acceptabilité et la faisabilité de l'intervention de rétroaction vidéo selon les infirmières.
Lorsque questionnées sur les facilitateurs et les barrières à l'utilisation de l'approche rétroaction vidéo dans le contexte du suivi SIPPE (voir Tableau 6), les infirmières se sont montrées positives face à cette approche et favorables à son utilisation.

\section{Tableau 5}

Acceptabilité et faisabilité de la rétroaction vidéo selon les infirmières $(n=8)^{a}$

\begin{tabular}{|c|c|c|c|}
\hline & & $\begin{array}{c}\text { Groupe A } \\
\text { n (\%) }\end{array}$ & $\begin{array}{c}\text { Groupe B } \\
\text { n (\%) }\end{array}$ \\
\hline Intervention facile à réaliser & Tout à fait & $6(100)$ & $6(100)$ \\
\hline \multirow{2}{*}{$\begin{array}{l}\text { La préparation et la réalisation de la vidéo s'intègrent bien à } \\
\text { mes tâches }\end{array}$} & Tout à fait & $0(0)$ & $2(33,3)$ \\
\hline & Plutôt & $2(33,3)$ & $4(66,7)$ \\
\hline \multirow{3}{*}{$\begin{array}{l}\text { Mon supérieur hiérarchique facilite l'intégration de ce type } \\
\text { d'intervention }\end{array}$} & Tout à fait & $0(0)$ & $4(66,7)$ \\
\hline & Plutôt & $1(16,7)$ & $2(33,3)$ \\
\hline & Moyennement/peu & $5(83,3)$ & $0(0)$ \\
\hline \multirow[t]{3}{*}{ J'ai participé pour faire plaisir à l'équipe de recherche } & Tout à fait/plutôt & $2(33,3)$ & $3(50,0)$ \\
\hline & Moyennement/peu & $3(50,0)$ & $2(33,3)$ \\
\hline & Pas du tout & $1(16,7)$ & $1(16,7)$ \\
\hline \multirow[t]{2}{*}{ Je suis satisfaite de mon attitude lors de l'intervention } & Tout à fait & $2(33,3)$ & $1(16,7)$ \\
\hline & Plutôt & $4(66,6)$ & $4(66,7)$ \\
\hline \multirow[t]{2}{*}{ Je recommanderais cette intervention aux infirmières } & Tout à fait & $2(33,3)$ & $5(83,3)$ \\
\hline & Plutôt & $3(50,0)$ & $1(16,7)$ \\
\hline \multirow[t]{3}{*}{ Facile de proposer cette intervention aux mères } & Tout à fait & $0(0)$ & $1(16,7)$ \\
\hline & Plutôt & $3(50,0)$ & $4(66,7)$ \\
\hline & Moyennement & $3(50,0)$ & $1(16,7)$ \\
\hline \multirow{2}{*}{$\begin{array}{l}\text { J'ai eu le soutien nécessaire pour préparer et réaliser } \\
\text { l'intervention }\end{array}$} & Tout à fait & $2(33,3)$ & $4(66,7)$ \\
\hline & Plutôt & $4(66,7)$ & $2(33,3)$ \\
\hline
\end{tabular}

a Quatre infirmières ont fait des interventions dans le groupe A et le groupe B.

\section{Tableau 6}

Facilitateurs et barrières à la rétroaction vidéo selon les infirmières $(n=8)$

\begin{tabular}{|c|c|}
\hline Facilitateurs & Barrières \\
\hline $\begin{array}{ll}\text { - } & \text { Domicile du parent adapté (espace, calme, être seule } \\
\text { avec l'infirmière) } \\
\text { - } \quad \text { Équipement technique facile d'utilisation } \\
\text { - } \quad \text { Répétition avec une même mère } \\
\text { - } \quad \text { Aspect positif de l'intervention ; mise sur les forces de } \\
\text { la mère, valorisant } \\
\text { - } \quad \text { Effet de complicité entre la mère et l'infirmière }\end{array}$ & $\begin{array}{ll}\text { - } & \text { Présence d'autres membres de la famille } \\
\text { - } & \text { Défis techniques de filmer durant l'allaitement } \\
\text { - } & \text { Manque de temps } \\
\text { - } \quad \text { Initiation des interactions limitée de la part des } \\
\text { bébés }\end{array}$ \\
\hline
\end{tabular}


Un des effets les plus positifs a été le sentiment de complicité avec la mère dans le cadre de cette intervention. Les infirmières valorisent l'aspect positif de l'intervention soit le fait qu'elle est centrée sur les interactions sensibles entre la mère et son enfant. Pour plusieurs mères, il semble que les occasions de valorisation de leurs capacités parentales soient rares. La valorisation semble avoir été appréciée autant par les infirmières que par les mères. Parmi les barrières à l'utilisation de l'approche, la plus fréquemment mentionnée a été la présence d'autres membres de la famille (conjoint, sœur, belle-mère, autre enfant), créant ainsi un malaise durant l'interaction filmée de même qu'au moment du retour. De plus, $83 \%$ d'entre elles estiment que l'approche est moyennement ou peu facilitée par leur supérieur hiérarchique.

\section{EFFETS DE L'INTERVENTION SUR LA SENSIBILITÉ MATERNELLE ET LA DURÉE DE L'ALLAITEMENT MATERNEL}

Un objectif spécifique de l'étude visait à estimer les effets préliminaires de la RV sur la sensibilité maternelle ainsi que sur la poursuite ou non de l'allaitement à trois mois. Le Tableau 7 suggère qu'il n'y a pas d'augmentation significative de la sensibilité après l'intervention, de même qu'en fonction des groupes. Un léger déclin dans le score est observé au temps 2 , ce qui est attendu considérant que la sensibilité s'améliore avec le temps et que plus le score est bas, plus la sensibilité est élevée. Le Tableau 8, pour sa part, suggère une légère tendance à la poursuite de l'allaitement à trois mois pour les mères des groupes expérimentaux en comparaison à celles du groupe témoin (Groupe A = $100 \%$, Groupe B = 83,3 $\%$ vs Groupe C $=50 \%$ ).

\section{Tableau 7}

Scores de sensibilité maternelle en fonction des groupes

\begin{tabular}{lcccc}
\hline & $\begin{array}{c}\text { Groupe A } \\
(\mathbf{n}=6)\end{array}$ & $\begin{array}{c}\text { Groupe B } \\
(\mathbf{n}=6)\end{array}$ & $\begin{array}{c}\text { Groupe A+B } \\
(\mathbf{n}=12)\end{array}$ & $\begin{array}{c}\text { Groupe C } \\
(\mathbf{n}=8)^{*}\end{array}$ \\
\hline Score total & & & & \\
Pré intervention & $1,3(1,1-1,8)$ & $1,8(1,5-2,0)$ & $1,6(1,2-1,8)$ & $1,4(1,3-1,7)$ \\
Post intervention & $1,1(1,1-1,3)$ & $1,3(1,2-1,6)$ & $1,2(1,1-1,5)$ & $1,2(1,2-1,4)$ \\
\hline
\end{tabular}

* Une donnée manquante.

Tableau 8

Poursuite de l'allaitement maternel à trois mois en fonction des groupes

\begin{tabular}{lccc}
\hline & Groupe A & Groupe B & Groupe C \\
& $\mathbf{n}(\%)$ & $\mathbf{n}(\%)$ & $\mathbf{n}(\%)$ \\
\hline Allaitement à 3 mois & $6(100)$ & $5(83,3)$ & $3(50,0) *$ \\
\hline
\end{tabular}

* Deux données manquantes.

DISCUSSION

Les résultats obtenus dans le cadre de cette étude confirment que la rétroaction vidéo combinée à de l'information sur l'attachement parents-enfant constitue une intervention faisable et acceptable du point de vue des mères et des infirmières. Les seuils fixés à $80 \%$ pour les critères d'acceptabilité et de faisabilité ont été atteints, sauf pour la perception de l'utilité de la rétroaction de l'infirmière pour les mères ayant reçu une seule intervention. Ce résultat tend à confirmer les données actuelles qui soutiennent que cette approche gagne à être utilisée à court terme, entre deux et huit fois pour obtenir des résultats optimaux (Bakermans-Kranenburg et al., 2003; Moss et al., 2018). Dans le cadre d'une prochaine 
étude, le nombre d'interventions pourrait varier entre deux et huit, tout en poursuivant l'analyse des différences perçues entre les groupes de manière à documenter le minimum d'interventions requises pour observer un résultat positif.

Le recrutement d'une clientèle vivant en contextes de vulnérabilité à qui l'on demande d'être filmée en contexte d'allaitement était un défi. D'ailleurs, six mères sur 29 ont refusé I'intervention, dont trois explicitement à cause du contexte d'allaitement. Toutefois, il s'agit d'un taux de participation élevé, probablement en partie attribuable au fait que l'intervention allait être réalisée par l'infirmière avec qui la mère avait déjà développé un lien de confiance dans le cadre du suivi SIPPE en période prénatale.

La rétroaction vidéo réalisée en contexte d'allaitement combinée à l'approche éducative sur la relation mère-enfant a le mérite d'être novatrice puisqu'elle permet de potentialiser les effets d'un soutien relationnel et éducatif. Autrement dit, en plus d'aider la mère à composer avec les défis de l'allaitement, cette approche semble avoir favorisé une meilleure connaissance de l'enfant par sa mère en misant sur l'observation des interactions de même qu'à un renforcement du sentiment de compétence à cause de la rétroaction positive de l'infirmière. Ces bienfaits attendus de la rétroaction vidéo ont été documentés, notamment dans les études de Tarabulsy et ses collaborateurs (2018) et de Juffer et ses collègues (2018). La présente étude ne permet pas de généraliser l'atteinte de ces bienfaits pour toutes les mères, mais suggère qu'une étude de plus grande envergure pourrait permettre de mieux comprendre comment utiliser l'approche et qui sont les mères le plus susceptibles d'en bénéficier.

Pour leur part, les infirmières sont favorables à l'utilisation de l'approche auprès de leur clientèle SIPPE. Il s'agit d'un résultat fort intéressant, surtout considérant qu'il s'agit d'une nouvelle approche, qui demande du temps, un équipement technique et une collaboration pour l'étude de la bande vidéo et la préparation du retour auprès de la mère. Les infirmières recommandent que la RV soit intégrée au suivi SIPPE afin d'en faciliter et d'en généraliser l'utilisation, et que les ressources nécessaires soient disponibles. Le fait que les infirmières participantes avaient suivi une formation sur l'attachement parents-enfant et la rétroaction vidéo de même que des séances de supervision clinique sur cette approche (variant de une à quatre), a fort probablement influencé le taux de participation. Conséquemment, la présente étude ne permet pas de généraliser les résultats aux infirmières d'autres CSSS. La présente étude est novatrice en ce qui concerne le contexte d'allaitement dans lequel la rétroaction vidéo a été utilisée. L'approche préconisée en contexte d'allaitement se situe dans une perspective de promotion de l'établissement de la relation mèreenfant et de prévention des difficultés. Elle a également le potentiel de favoriser la poursuite de l'allaitement, considérant la relation entre la qualité de la relation mère-enfant et la durée de l'allaitement (Britton et al., 2006). Cette perspective positive et prometteuse valait la peine d'être testée et les résultats permettent de croire que des bienfaits sur la relation peuvent être observés dès les premiers mois de la vie de l'enfant, particulièrement en contexte de vulnérabilité.

Les effets de la rétroaction vidéo sur la sensibilité maternelle n'ont pu être détectés avec le présent devis. Bakermans-Kranenberg et leurs collègues (2003) suggèrent qu'environ cinq interventions sont nécessaires pour obtenir un résultat mesurable sur la qualité de la sensibilité maternelle, alors que notre étude a testé l'impact d'une et deux interventions. Une étude permettant d'atteindre la dose d'interventions recommandées est nécessaire pour documenter l'effet de la RV sur la sensibilité maternelle en contexte d'allaitement. En outre, un échantillon plus grand de même que l'utilisation concomitante d'un outil d'observation de la qualité des interactions entre la mère et son enfant sont à considérer dans une prochaine étude. Dans la même lignée, on ne peut pas conclure à un effet de l'intervention sur la poursuite de l'allaitement maternel à trois mois. Les écrits étant assez unanimes pour proposer une relation positive entre la sensibilité maternelle et la poursuite de l'allaitement (Britton et al., 2006; Jansen et al., 2008; Peñacoba et Catala, 2019), on peut espérer trouver ce lien positif à l'aide d'un devis expérimental. 


\section{LIMITES ET FORCES}

La principale limite de la présente étude est le petit nombre de mères par groupe. Quelques mères de plus dans chaque groupe auraient peutêtre pu permettre de mieux décrire la différence de l'effet perçu entre une et deux interventions du point de vue des mères. Cependant, nous ne croyons pas que l'effet sur les scores de sensibilité et la poursuite de l'allaitement en auraient été modifiés. Le fait que les infirmières participant à l'étude avaient préalablement suivi une formation sur l'attachement parents-enfant et la rétroaction vidéo limite certainement la généralisation des résultats à d'autres contextes cliniques. Enfin, puisqu'une ou deux interventions ont été réalisées dans le cadre de la présente étude pilote alors qu'autour de cinq interventions sont nécessaires pour obtenir un effet mesurable sur la sensibilité maternelle, on ne peut pas conclure à l'efficacité de l'approche. La principale force de l'étude est l'utilisation d'un essai clinique randomisé pragmatique ayant favorisé le recrutement des mères ainsi que l'acceptabilité et la faisabilité de

I'intervention. Le devis pragmatique est proche de la pratique clinique puisque l'intervention à évaluer est adaptée à la réalité du terrain (Oxman et al., 2009; Zwarenstein et Treweek, 2009). Par ailleurs, il s'agit de la première étude à évaluer I'utilisation de la rétroaction vidéo en contexte d'allaitement et avec des infirmières. Le fait que l'intervention réalisée se situe dans une perspective de promotion de la relation et de prévention des difficultés la place dans une rare catégorie dans ce domaine où l'intervention est utilisée principalement dans une perspective de traitement des problèmes relationnels, voire même dans un contexte de maltraitance (Moss et al., 2011). Soutenir l'établissement de la relation mère-enfant dès la naissance est certainement une intervention que l'infirmière peut réaliser, avec les connaissances et compétences nécessaires, surtout si elle se situe en contexte d'allaitement.

\section{CONCLUSION}

La présente étude soutient l'acceptabilité et la faisabilité d'une intervention de rétroaction vidéo durant l'allaitement combinée à de l'information sur l'attachement parents-enfant auprès de mères vivant en contextes de vulnérabilité. L'approche a obtenu les effets escomptés en termes de participation des mères et des infirmières et le volet qualitatif de l'étude soutient les bienfaits de cette approche sur la relation mère-enfant. Un effet obtenu, mais non anticipé, est celui de renforcer la relation de confiance entre la mère et l'infirmière, ce qui constitue un gain important puisque la relation de confiance constitue la composante la plus importante pour assurer l'atteinte des objectifs d'intervention du programme SIPPE. Considérant qu'il s'agit d'une approche exigeante en termes de temps et de ressources, mais payante sur le plan des bienfaits, il est recommandé de poursuivre les études dans la perspective de bien cibler les mères les plus susceptibles d'en bénéficier ainsi que le nombre de séances nécessaires pour obtenir un résultat positif sur la qualité de la relation.

Contribution des auteurs : LB, ASP, ML et $\mathrm{MH}$ ont conçu l'étude et révisé et approuvé la version finale du manuscrit. LB et ML ont supervisé la collecte et l'analyse des données. ASP a réalisé la collecte des données. $L B, A S P$ et $M L$ ont organisé l'article et rédigé la première ébauche.

Remerciements : Les auteurs remercient les mères et les infirmières du CSSS-IUGS pour leur participation à l'étude ainsi que Andrée Fontaine, Guadalupe Puentes-Neuman et Yves Lajoie de l'Équipe régionale de formation en attachement parent-enfant. Les auteurs remercient également la Pre Nancy Feeley pour sa contribution à la conception de la méthodologie de l'étude.

Sources de financements : Les auteurs ont reçu du financement de Réseau de recherche en interventions en sciences infirmières du Québec (RRISIQ) pour la réalisation de cette étude.

Déclaration de conflits d'intérêts : Les auteurs déclarent qu'il n'y a pas de conflits d'intérêts.

Numéro du certificat d'éthique : L'étude a reçu l'approbation éthique du Comité d'éthique de la recherche du Centre de santé et de services sociaux - Institut universitaire de gériatrie de Sherbrooke (\# 2012-14/BELL).

Reçu/Received: 6 Jan 2019 Publié/Published: 29 Juin 2020 


\section{RÉFÉRENCES}

Bakermans-Kranenburg, M. J., van IJzendoorn, M. H., \& Juffer, F. (2003). Less is more: Meta-analyses of sensitivity and attachment interventions in early childhood. Psychological Bulletin, 129, 195-215. https://doi.org/10.1037/00332909.129.2.195

Bell, L. (2008). L'établissement de la relation parents-enfant. Un modèle d'évaluation et d'intervention ayant pour cible la sensibilité parentale. L'infirmière clinicienne, 5(2), 39-44.

Bell, L., Goulet, C., St-Cyr Tribble, D., Paul, D., \& Polomeno, V. (1996). Une analyse du concept d'attachement parents-enfant. Recherche en soins infirmiers, 46, 4-13.

Bell, L., Goulet, C., St-Cyr Tribble, D., \& Paul, D. (2004a). L'Échelle de sensibilité maternelle. Document inédit. Université de Sherbrooke.

Bell, L., Goulet, C., St-Cyr Tribble, C., \& Paul, D. (2004b). Établissement du lien mère-enfant et père-enfant à la période périnatale. Perspective infirmière, juillet-août, 13-22.

Bell, L., Lacombe, M., Gallagher, F., Ferland, M., \& Couture, M.E. (2012). Les facteurs impliqués dans l'arrêt précoce de l'allaitement maternel. Soins Pédiatrie-Puériculture, 269, 39-45.

Bell, L., St-Cyr Tribble, D., Goulet, C., Paul, D., \& Tronick, E. Z. (2007). Mother's and father's early relationship with their infant : Similar yet temporally discordant themes. Canadian Journal of Midwifery Research and Practice, 6(3), 30-41.

Bowlby, J. (1984). Attachment and Loss. Vol. 1: Attachment (Revised Ed.). Penguin Books.

Britton, J. R., Britton, H. L., \& Gronwaldt V. (2006). Breastfeeding, sensitivity, and attachment. Pediatrics, 118(5):e1436-e1443.

Cassidy, J. (1999). The nature of the child's ties. In J. Cassidy \& P. R. Shaver (Eds.), Handbook of Attachment: Theory, Research, and Clinical Applications (pp. 3-20). Guilford Press.

Courtois, E., Lacombe, M., \& Tysio, D. (2012). Les facteurs prédictifs de la poursuite de l'allaitement maternel jusqu'à six mois de vie du nourrisson, en région parisienne. $5^{e}$ Congrès mondial des infirmières et des infirmiers francophones. Genève, Suisse.

Couture, M. E., Lacombe, M., \& Bell, L. (sous presse). Sensibilité maternelle en contexte d'allaitement. Perspective infirmière.

De Wolff, M. S. \& Van IJzendoorn, M. H. (1997). Sensitivity and attachment: A meta-analysis on parental antecedents of infant attachment. Child Development, 68, 571-591.

Doyon, M. (2010). Étude pilote d'un programme préventif d'exercices physiques sur les symptômes dépressifs post-partum et la sensibilité maternelle [mémoire de maîtrise inédit]. Université de Sherbrooke.

Dubé, M. M., Bell, L., \& Lacombe, M. (2019). Étude à devis mixte de la perception des mères sur l'établissement de la relation mère-bébé en contexte d'allaitement difficile ayant mené au sevrage [document soumis pour publication]. Revue francophone internationale de recherche infirmière.

Firk, C., Dahmen, B., Lehmann, C., Niessen, A., Koslowski, J., Rauch, G., Schwarte, R., Stich, K., Konrad, K., \& Herpertz-Dahlmann, B. (2015). A mother-child intervention program in adolescent mothers and their children to improve maternal sensitivity, child responsiveness and child development (the TeeMo study): study protocol for a randomized controlled trial. Bio med central (BMC), 16, p. 230. https://doi.org/10.1186/s13063-015-0747-5

Fukkink, R. G. (2008). Video feedback in widescreen: A meta-analysis of family programs. Clinical Psychology Review, 28, 904916. https://doi.org/10.1016/j.cpr.2008.01.003

Hodes, M. W., Meppelder, M., de Moor, M., Kef, S., \& Schuengel, C. (2018). Effects of video-feedback intervention on harmonious parent-child interaction and sensitive discipline of parents with intellectual disabilities: A randomized controlled trial. Child Health Care Developpement, 44(2), 304-311. https://doi.org/10.1111/cch.12506

Hoffenkamp, H. N., Tooten, A., Hall, R. A., Braeken, J., Eliëns, M. P., Vingerhoets, A. J, \& van Bakel, H. J., (2015). Effectiveness of hospital-based video interaction guidance on parental interactive behavior, bonding, and stress after preterm birth: A randomized controlled trial. Journal of Consulting and Clinical Psycholgy, 83(2), pp. 416-29. https://doi.org/10.1037/a0038401

lles, J.E., Rosan, C., Wilkinson, E., Ramchandani P. G. (2017). Adapting and developing a video-feedback intervention for coparents of infants at risk of externalising behaviour problems: A feasibility study. Clinical Child Psychologic Psychiatric, 22(3), 483-499. https://doi.org/0.1177/1359104517704025

Jansen, J., de Weerth C., \&, Riksen-Walraven J. M. (2008). Breastfeeding and the mother-infant relationship: A review. Developmental Review, 28, 503-521. https://doi.org/10.1016/j.dr.2008.07.001

Juffer, F., Bakermans-Kranenburg, M. J., \& Van IJzendoorn, M. H. (2008). Promoting positive parenting: An attachment-based intervention. Taylor and Francis Group.

Juffer, F., \& Bakermans-Kranenburg, M. J. (2018). Working with video-feedback intervention to promote positive parenting and sensitive discipline (VIPP-SD): A case study. Journal of Clinical Psychology, 74(8), 1346-1357. https://doi.org/10.1002/jclp.22645 
Kronborg, H., Harder, I., \& Hall, E. O. (2015). First time mothers' experiences of breastfeeding their newborn. Sexual and Reproductive Health Care Journal, 6, 82-87. https://doi.org/10.1016/j.srhc.2014.08.004

Larone Juneau, A. (2013). Intervention éducationnelle de massage auprès de parents de nouveau-nés prématurés dans le but de favoriser leur sensibilité parentale [mémoire de maîtrise inédit]. Université de Montréal.

Lavallée, A., Aita, M., Côté, J., Bell, L., \& Luu, T. M. (2019). Guided Participation intervention for mothers in their preterm infant's therapeutic POSITioning and care (GP_Posit) for maternal sensitivity and infant neurodevelopment: Pilot randomized trial protocol [document soumis pour publication]. Nursing Research.

Leclère, C., Viaux, S., Rabain, D., Khun-Franck, L., Dubois, C., Camon-Senechal, L., Couetoux-Jungman, F., Cohen, D., \& Missonnier, S. (2018). Video feedback in situation of emotional neglect: A tool for assessment and care of early interactions? Annales Médico-Psychologiques, 176(3), 296-300. https://doi.org/10.1016/j.amp.2018.01.003

L'Écuyer, R. (1990). Méthodologie de l'analyse développementale de contenu : méthode GPS et concept de soi. Presses de I'Université du Québec.

Martel, M. J., St-Cyr Tribble, D., \& Bell, L. (2011). L'établissement de la relation parent-enfant prématuré à l'unité néonatale. Revue québécoise de psychologie, 32(2), 11-36.

Ministère de la Santé et des Services sociaux du Québec (2019). Les services intégrés en périnatalité pour la petite enfance à I'intention familles vivant en contexte de vulnérabilité. https://publications.msss.gouv.qc.ca/msss/fichiers/2018/18-836-02W.pdf

Moss, E., Dubois-Comtois, K., Cyr, C., Tarabulsy, G., St-Laurent, D., \& Bernier, A. (2011). Efficacy of a home-visiting intervention aimed at improving maternal sensitivity, child attachment, and behavioral outcomes for maltreated children: $\mathrm{A}$ randomized control trial. Development \& Psychopathology, 23, $195-210$. https://doi.org/10.1017/S0954579410000738

Moss, E., Tarabulsy, G., Dubois Comtois, K., Cyr, C., Bernier, A., St Laurent, D. (2018). The attachment video-feedback intervention program: development and validation. Dans Steele, H. et M. Steele (dir.), Handbook of attachment-based interventions (p. 318-338). Guilford Press.

Oxman, A. D., Lombard, C., Treweek, S., Gagnier, J. J., Maclure, M., \& Zwarenstein, M. (2009). Why we will remain pragmatists: Four problems with the impractical mechanistic framework and a better solution. Journal of Clinical Epidemiology, 62, 485-488.

Peñacoba, C., \& Catala, P. (2019). Associations between breastfeeding and mother-infant relationships : A systematic review. Breastfeeding Medicine, 14(9), 616-629. https://doi.org/10.1089/bfm.2019.0106

Poslawsky, I. E., Naber, F. B., Bakermans-Kranenburg, M. J., van Daalen, E., van Engeland, H., van IJzendoorn, M. H. (2014). Video-feedback intervention to promote positive parenting adapted to autism (VIPP-AUTI): A randomized controlled trial. Autism, 19, 588-603. https://doi.org/10.1177/1362361314537124

Schoemaker, N. K., Jagersma, G., Stoltenborgh, M., Maras, A., Vermeer, H.J., Jufer, F., \& Alink, L.R.A. (2018). The effectiveness of video-feedback intervention to promote positive parenting for foster care (VIPP-FC): study protocol for a randomized controlled trial. BMC Psychol, 6(38). https://doi.org/10.1186/s40359-018-0246-z

St-Laurent, D., Larin, S., Tarabuslsy, G. M., Moss, E., Bernier, A., Dubois-Comtois, K., \& Cyr, C. (2008). Intervenir auprès de familles vulnérables selon les principes de la théorie de l'attachement. L'infirmière clinicienne, 5(8), 21-29.

Stein, A., Woolley, H., Senior, R., Hertzmann, L., Lovel, M., Lee, Cooper, S., Wheatcroft, R., Challacombe, F., Patel, P., NicolHarper, R., Menzed, P., Fairburn, C. G., Schmidt, A., Juszczak, E., \& Fairburn, G. (2006). Treating disturbances in the relationship between mothers with bulimic eating disorders and their infants: A randomized, controlled trial of video feedback. American Journal of Psychiatry, 163, 899-906.

Tharner, A., Luijk, M. P., Raat, H., IJzendoorn, M. H., Bakermans-Kranenburg, M. J., Moll, H., Jaddoe, V.W., Hofman, A., Verhulst, F.C., \& Tiemeier, H. (2012). Breastfeeding and its relation to maternal sensitivity and infant attachment. Journal of Developmental and Behavioral Pediatrics, 33, 396-404. https://doi.org/10.1097/DBP.0b013e318257fac3

Tarabulsy, G. M., Dubois-Comtois, K., Baudry, C., Moss, E., Roberge, A., Savage, L.E., Cyr, C., St-Laurent, D., \& Bernier, A. (2018). A case study illustrating the use of an attachment video-feedback intervention strategy. Journal of Clinical Psychology, 74(8), 1358-1369. https://doi.org/10.1002/jclp.22646

Turner, S., Mayumi Maruyama, J., Matijasevich, A., \& Pastor-Valero, M. (2019). Breastfeeding and the development of socioemotional competencies : A systematic review. Breastfeeding Medicine, 14(10), 1-14. https://doi.org/10.1089/bfm.2019.0147

Yagmur, S., Mesman, J., Malda, M., Bakermans-Kranenburg, M. J., \& Ekmekci, H. (2014). Videofeedback intervention increases sensitive parenting in ethnic minority mothers: A randomized control trial. Attachment \& Human Development, 16, 371-386.

Zelenko, M., \& Benham, A. (2000). Videotaping as a therapeutic tool in psychodynamic infant-parent therapy. Infant Mental Health Journal, 21(3), 192-203. https://doi.org/10.1002/1097-0355(200007)21:3<192::AID-IMHJ4>3.0.CO;2-\%23

Zwarenstein, M., \& Treweek, S. (2009). What kind of randomized trials do we need? Canadian Medical Association Journal, 180(10), 998-1000. 